\title{
$\mu$ Herculis: Analysis of EW time series of a solar-type pulsator
}

\author{
E. Carolo, ${ }^{1}$ R. Claudi, ${ }^{1}$ S. Benatti, ${ }^{2}$ and A. Bonanno ${ }^{3}$ \\ 1 INAF - Osservatorio Astronomico di Padova, Vicolo Osservatorio 5, 35122, Padova, Italy \\ ${ }^{2}$ CISAS, Universitá degli Studi di Padova, via Venezia 15, 35131, Padova, Italy \\ 3 INAF - Osservatorio Astrofisico di Catania, via S. Sofia 78, 95123, Catania, Italy
}

\begin{abstract}
The G5 subgiant star $\mu$ Herculis was observed in June 2006 by means of the high-resolution spectrograph SARG operating at the $3.6 \mathrm{~m}$ Italian telescope TNG (Telescopio Nazionale Galileo) at the Canary Islands, exploiting the iodine cell technique. A time series of about 1200 spectra was acquired during 7 observing nights. Data analysis of the radial velocity time series (Bonanno et al. 2008) has shown a significant power excess centred at $1.2 \mathrm{mHz}$, with $\sim 0.9 \mathrm{~ms}^{-1}$ peak amplitude. Here we present an analysis of the Equivalent Width time series of $\mathrm{H}_{\alpha}$ and other lines.
\end{abstract}

Individual Objects: $\mu$ Her

\section{Method}

We considered the red part of echelle spectrum of $\mu$ Her (between 600 and $900 \mathrm{~nm}$ ), since it is insensitive to the iodine cell. This allows us to use the Equivalent Width (EW) variations to evaluate the presence of stellar pulsations and granulation. The analyzed lines were: $\mathrm{FeI}$ $(\lambda=6393.2,6545.8,6677.6 \AA ̊), \mathrm{NiI}(6643.25 \AA)$ and $\mathrm{H}_{\alpha}(6563 \AA)$. The $\mathrm{H}_{\alpha}$ line is on the rim of two adjacent spectral orders of the echelle spectrum. In order to have the red and blue wings of the line both on the same spectrum, we merged the two parts. To do this in a safe way, we correct the two orders for the continuum before merging them. For all the lines we evaluate the EW in the usual way, using the following expression:

$$
E W=\int_{\lambda_{1}}^{\lambda_{2}}\left(1-R_{\lambda}\right) d \lambda, \text { where } R_{\lambda}=\frac{f(\lambda)}{f(c)}
$$

\section{Time series and power spectra of EWs}

Figure 1 shows the EW time series of one of the analyzed lines $\left(H_{\alpha}\right)$. The observations were conducted on 7 nights in June 2006 using SARG, the high resolution spectrograph of the TNG. As expected the computed power spectrum of the EW time series shows a very low amplitude. Figure 2 shows as example the $\mathrm{H}_{\alpha}$ Power Spectrum.

The power spectrum does not show evidence of a power excess due to pulsation. Nevertheless a concentration of power is present at low frequencies. This feature is similar to the background power found by Kjeldsen et al. (1999) for $\alpha$ Cen A and by Leccia et al. (2007) for Procyon A. These authors hypothesized this noise to be due to stellar granulation.

A more detailed analysis of the time series of EWs and the estimate of the stellar granulation using by power density spectrum is in progress. 
EW time series



Figure 1: Time series of EWs of $\mathrm{H}_{\alpha}$.



Figure 2: Power spectra of EWs of $\mathrm{H}_{\alpha}$.

\section{References}

Bonanno, A., Benatti, S., Claudi, R., et al. 2008, ApJ, 676, 1248

Kjeldsen, H., Bedding, T. R., Frandsen, S., \& Dall, T. H. 1999, MNRAS, 303, 579

Leccia, S., Kjeldsen, H., Bonanno, A., et al. 2007, A\&A, 464, 1059 